\title{
Competencias del Profesor de Educación Primaria
}

\author{
José Luis Lupiáñez' \\ 'Universidad de Granada - Espanha
}

RESUMEN - Competencias del Profesor de Educación Primaria. En este documento recojo diferentes acercamientos al establecimiento de las competencias que deberían desarrollar los profesores de matemáticas de Educación Primaria. Para ello, en primer lugar analizo el papel de la noción de competencia en el marco de la formación de profesores. A continuación me centro en las directrices europeas que se han empleado para el diseño de las nuevas titulaciones dirigidas a la formación de maestros, prestando especial atención al caso español. Finalmente, analizo diferentes trabajos en investigaciones centradas en el área de matemáticas.

Términos-clave: Competencias. Formación de Profesores. Educación Primaria.

ABSTRACT - Competences of the Elementary School Teacher. In this document I collect various approaches to the establishment of the competences that mathematics teachers should develop in Elementary School. To do this, first I analyze the role of the notion of competence as part of teachers' education. Next, I focus on the European guidelines that have been used for the design of new qualifications aimed at the teachers' education, with special attention to the Spanish case. Finally, I analyze different papers in research focused on the area of mathematics.

Keywords: Competences. Teachers' Education. Elementary School.

Educação \& Realidade, Porto Alegre, v. 39, n. 4, p. 1089-1111, out./dez. 2014.1089 Disponível em: <http://www.ufrgs.br/edu_realidade> 
En la formación inicial de profesores de matemáticas, existe una constatada preocupación por establecer el perfil profesional de los futuros profesores en términos de las competencias que deberían desarrollar estos para el ejercicio de su actividad docente (Abbott; Huddleston, 2000; Beck; Hart; Kosnik, 2002; Gómez, 2007; Niss, 2004a; 2004b; Recio, 2004; Rico, 2004).

Son diversas las instituciones, programas y grupos de investigación que se han ocupado recientemente de las competencias profesionales del profesor en formación, que han proporcionado información confiable. Sin embargo, todavía existen imprecisiones y lagunas acerca de cuestiones fundamentales como cuál es la estructura y jerarquía de esas competencias y cómo puede optimizarse su desarrollo en un programa de formación (Lupiáñez, 2009).

Estas limitaciones se constatan en distintos autores. Gómez (2007, p. 123) destaca “[...] la carencia, por el momento, de una caracterización suficientemente detallada de las competencias del profesor, en general, y del profesor de matemáticas en particular". Oser, Achtenhagen y Renold (2006, p. 7) constatan que, del mismo modo que no existe una teoría final de la instrucción y a pesar de que existen propuestas específicas de formación de profesores basadas en competencias, “[...] todavía vamos hacia una teoría sobre las competencias profesionales del profesor".

En este trabajo nos centramos en la selección y organización de las competencias profesionales que tiene que desarrollar el profesor de matemáticas de primaria en un programa de formación inicial. La caracterización de la noción de competencia que asumimos es la propuesta por Rico y Lupiáñez (2008, p. 71-74), en torno a tres componentes: unos conocimientos que pueden provenir de diferentes disciplinas, determinadas capacidades que movilizan esos conocimientos y una serie de contextos, en los que esas capacidades se ponen en juego con un determinado propósito.

En primer lugar concretaremos más específicamente esas tres componentes para, a continuación, describir y justificar algunas propuestas de listado de competencias que deberían desarrollar los profesores de matemáticas en formación.

\section{Diferentes Aproximaciones a las Competencias Específicas del Profesor}

Desde la investigación en Didáctica de la Matemática se ha tratado de establecer y organizar las competencias que deben desarrollar los profesores en un programa de formación. De todos ellos, destacamos algunas aportaciones con objeto de delimitar más concretamente los componentes de esas competencias.

Achtenhagen, Oser y Renold (2006, p. 297), relacionan las competencias profesionales del profesor con aquellas situaciones de enseñan-

1090 Educação \& Realidade, Porto Alegre, v. 39, n. 4, p. 1089-1111, out./dez. 2014. Disponível em: <http://www.ufrgs.br/edu_realidade> 
za y aprendizaje en las que un profesor tiene que intervenir: "La relación entre el desarrollo de competencias por un lado, y el dominio de situaciones de enseñanza y aprendizaje por otro, constituye un viejo problema que aborda la cuestión central de la formación de profesores". Desarrollar competencias para un profesor implica, para cada una de esas situaciones, una parte de diagnóstico, una de actuación y finalmente una de valoración. De este modo destacan que el desarrollo de competencias profesionales docentes es un conocimiento situado.

Estos autores, basándose en investigaciones y proyectos previos ${ }^{1}$, señalan que la noción de competencia profesional del profesor se conceptualiza en torno a cuatro dimensiones: una competencia personal o autocompetencia, que incluye diferentes aspectos del desarrollo personal profesional como cognición, emoción, motivación, metacognición o ética; una competencia cognitiva y del conocimiento del contenido, que incluye requerimientos teóricos y analíticos como para, por ejemplo, "aplicar conceptos"; una competencia funcional y didáctica que comprende requerimientos técnicos, prácticos y funcionales, como para, por ejemplo, "usar herramientas, equipamiento y recursos técnicos"; y una competencia social y comunicativa que incluye requerimientos interpersonales, para, por ejemplo, "interactuar en servicio de otros, y con otros" (Achtenhagen; Oser; Renold, 2006, p. 297).

Al contemplar las competencias profesionales del profesor en formación dentro de este marco, el conocimiento disciplinar requerido debiera ser un conocimiento teórico y profesional sobre el currículo de Educación Primaria, en nuestro caso de las matemáticas escolares de este nivel educativo.

Gómez (2007) recoge otro referente sobre competencias del profesor que es importante. En Australia se trabajó en un esquema organizador de las competencias del profesor en torno a cinco dimensiones: facilitar el aprendizaje de los escolares, evaluar e informar sobre el resultado de ese aprendizaje, comprometerse en el aprendizaje profesional, participar en el diseño curricular y en otras iniciativas centradas en la obtención de resultados y asociarse con la comunidad escolar (Department of Education and Training, 2004). En este caso las dimensiones contemplan dos focos de atención. En primer término destacan dos aspectos relativos al aprendizaje de los escolares; en segundo lugar consideran tres facetas del trabajo del profesor como miembro de una comunidad.

Finalmente, destacamos una reflexión de Claude Lessard, un experto en competencias canadiense, que señala solo dos dimensiones curriculares, pero que encontramos interesante ya que pone de manifiesto la complejidad de caracterizar las competencias relacionadas con disciplinas curriculares específicas (Luengo; Luzón; Torres, 2008, p. 6):

En el caso de la situación de la formación profesional del docente, todo el mundo comprende fácilmente la lógica

Educação \& Realidade, Porto Alegre, v. 39, n. 4, p. 1089-1111, out./dez. 2014.1091 Disponível em: <http://www.ufrgs.br/edu_realidade> 
de esa formación, un futuro docente debe aprender a saber gestionar una clase, eso es una competencia. Podemos pensar que en una situación de formación hay un perfil de salida de ese docente, que va a suministrar las herramientas adecuadas para gestionar la clase de manera competente. Yo no digo que sea fácil de hacer, pero comprendemos de qué se trata. Sin embargo, cuando se afirma, por ejemplo, que hay que tener la competencia matemática, no puedo resolver esa cuestión sin hacer referencia a lo que son las matemáticas, a su construcción histórica, a las grandes operaciones matemáticas, a su campo conceptual y tengo que construir situaciones de aprendizaje que permitan a los alumnos aprender para aprender esas operaciones, para integrar los diferentes campos conceptuales, para utilizar esos conceptos en diferentes contextos.

Como vemos en estas referencias, la noción de competencia o competencias del profesor se articula en torno a diferentes dimensiones y se describe con distintos niveles de precisión, dependiendo de los autores. Si analizamos estas aportaciones que los distintos investigadores considerados hacen en relación con las competencias que debe desarrollar un profesor de matemáticas para sistematizarlas desde una perspectiva curricular, es posible ubicar cada una de ellas según el esquema de dimensiones y niveles propuesto por Rico (1997b). Las dimensiones son las mismas ya establecidas en ese concepto de currículo, pero sin embargo, los distintos autores destacan diferentes focos prioritarios para establecer los niveles con los que concretan sus competencias (Lupiáñez, 2009, p. 151-152).

En cuanto a los conocimientos requeridos para que un profesor de matemáticas pueda desarrollar y lograr las competencias analizadas, podemos inferir con precisión que tales conocimientos son:

- la noción de currículo, articulada en sus diferentes dimensiones y niveles;

- herramientas de análisis de las matemáticas escolares, desde un punto de vista conceptual, cognitivo, formativo y social; y

- herramientas de diseño, puesta en práctica y evaluación de actividades de enseñanza y aprendizaje.

Por tanto, el profesor en formación conoce y emplea conceptos y procedimientos sobre las matemáticas escolares y otros conocimientos didácticos sobre su enseñanza y aprendizaje, que le permiten llevar a cabo el análisis didáctico de un tema de matemáticas, con objeto de diseñar y justificar actividades de enseñanza y aprendizaje (Gómez, 2007; Lupiáñez, 2009; Rico; Lupiáñez; Molina, 2013).

Desde esta caracterización, a continuación describimos diferentes acercamientos al establecimiento de las competencias que deben desarrollar los profesores en formación. Nos centramos en tres fuentes

1092 Educação \& Realidade, Porto Alegre, v. 39, n. 4, p. 1089-1111, out./dez. 2014 Disponível em: <http://www.ufrgs.br/edu_realidade> 
principales: aquellas que proceden de directrices europeas, las establecidas en el Grado de Maestro de Educación Primaria y, finalmente, dos listados de competencias específicamente centrados en Matemáticas.

\section{Directrices Europeas sobre las Competencias del Profesor en Formación}

La Recomendación del Parlamento y el Consejo de la Unión Europea a los Estados miembros ha tenido impacto en las directrices curriculares de los sistemas educativos de los países de la Unión y ha guiado su evolución. Este y otros documentos comunitarios consideran central la noción de competencia en su marco conceptual y en su normativa, de manera significativa para el currículo de la educación obligatoria. Diferentes proyectos e iniciativas europeas han ido dando forma a la expresión de las finalidades formativas en términos de competencias, como describen Rico y Lupiáñez (2008, p. 111-132).

Este marco de referencia formativo, que se detecta a nivel internacional como determinante de nuevas orientaciones educativas, fue destacado por la Comisión Internacional sobre la educación para el siglo XXI en su Informe a la UNESCO del año 1996 y, desde entonces, ha servido de acicate e impulsado las reformas curriculares y las nuevas directrices que se han derivado. En ese documento encontramos algunas de las bases conceptuales y políticas del marco de competencias. res: aprender a conocer, aprender a hacer, aprender a vivir juntos, aprender a ser.

Aprender a conocer, combinando una cultura general suficientemente amplia con la posibilidad de profundizar los conocimientos en un pequeño número de materias. Lo que supone, además, aprender a aprender para poder aprovechar las posibilidades que ofrece la educación a lo largo de la vida.

Aprender a hacer a fin de adquirir no solo una calificación profesional sino, más generalmente, una competencia que capacite al individuo para hacer frente a gran número de situaciones y trabajar en equipo. Pero, también, aprender a hacer en el marco de las distintas experiencias sociales o de trabajo que se ofrecen a los jóvenes y adolescentes, bien espontáneamente a causa del contexto social o nacional, bien formalmente gracias al desarrollo de la enseñanza por alternancia.

Aprender a vivir juntos desarrollando la comprensión del otro y la percepción de las formas de interdependencia realizar proyectos comunes y prepararse para tratar los conflictos- respetando los valores de pluralismo, comprensión mutua y paz.

Aprender a ser para que florezca mejor la propia personalidad y se esté en condiciones de obrar con creciente

Educação \& Realidade, Porto Alegre, v. 39, n. 4, p. 1089-1111, out./dez. 2014.1093 Disponível em: <http://www.ufrgs.br/edu_realidade> 
capacidad de autonomía, de juicio y de responsabilidad personal. Con tal fin, no menospreciar en la educación ninguna de las posibilidades de cada individuo; memoria, razonamiento, sentido estético, capacidades físicas, aptitudes para comunicar [...] (Delors, 1996, p. 109).

Quizás la iniciativa más ambiciosa a nivel europeo, centrado en la educación superior, es el establecimiento del Espacio Europeo de Educación Superior (EEES), que constituye un complejo plan de la Unión Europea para unificar criterios estructurales, organizativos y funcionales entre todas las universidades europeas. Aunque originalmente se concibió en la Declaración de la Sorbona (Allegre; Berlinguer; Blackstone; Rüttgers, 1998), la Declaración de Bolonia (Einem et al., 1999) fue la primera en la que se avanzó estructuralmente en este proyecto ${ }^{2}$.

Esta iniciativa, que en la actualidad tiene un impacto social considerable como se refleja en su gran repercusión mediática (Sotelo, 2009), tiene varias implicaciones y, entre ellas, destacamos el proyecto Sócrates-Erasmus Tuning Educational Structures in Europe, al que nos referiremos desde ahora como Tuning (González; Wagenaar, 2003; 2006).

El proyecto Tuning persigue la adopción de un sistema de titulaciones reconocibles y comparables en Europa, y recientemente se ha llevado a cabo un estudio similar en América Latina (Beneitone et al., 2007) ${ }^{3}$.

Una de sus aportaciones principales ha sido la puesta en uso del concepto de competencia para precisar el significado de los nuevos objetivos formativos de la educación universitaria:

Las competencias tienden a transmitir el significado de lo que la persona es capaz de o es competente para ejecutar, el grado de preparación, suficiencia o responsabilidad para ciertas tareas. [...] El concepto de las competencias trata de seguir un enfoque integrador, considerando las capacidades por medio de una dinámica combinación de atributos que juntos permiten un desempeño competente como parte del producto final de un proceso educativo [...]. Las competencias y las destrezas se entienden como conocer y comprender (conocimiento teórico de un campo académico, la capacidad de conocer y comprender), saber cómo actuar (la aplicación práctica y operativa del conocimiento a ciertas situaciones), y saber cómo ser (los valores como parte integrante de la forma de percibir a otros y vivir en un contexto social). Las competencias representan una combinación de atributos (con respecto al conocimiento y sus aplicaciones, aptitudes, destrezas y responsabilidades) que describen el nivel o grado de suficiencia con que una persona es capaz de desempeñarlos (González; Wagenaar, 2003, p. 69-70, en negritas en el original).

1094 Educação \& Realidade, Porto Alegre, v. 39, n. 4, p. 1089-1111, out./dez. 2014. Disponível em: <http://www.ufrgs.br/edu_realidade> 
La educación [universitaria] deberá centrarse en la adquisición de competencias por parte del estudiante. Se trata de centrar la educación en el estudiante. El papel fundamental del profesor debe ser el de ayudar al estudiante en el proceso de adquisición de competencias. El concepto de competencia pone el acento en los resultados del aprendizaje, en lo que el estudiante es capaz de hacer al término del proceso educativo y en los procedimientos que le permitirían continuar aprendiendo de forma autónoma a lo largo de su vida (Moreno; Bajo; Moya; Maldonado; Tudela, 2007, p. 1).

En el marco del proyecto Tuning se consideran dos tipos de competencias. Aquellas que se relacionan con áreas temáticas determinadas (como Geología, Historia o Matemáticas), y que se denominan específicas; y las competencias genéricas que recogen “[...] aquellos atributos compartidos que pudieran generarse en cualquier titulación y que son considerados importantes por ciertos grupos sociales" (González; Wagenaar, 2003, p. 70).

Estas nociones sobre competencias son coincidentes con la caracterización de competencia que acotamos anteriormente, en la que destacan unos componentes cognitivos, una serie de finalidades asignadas y un contexto en el que se desempeña la competencia. La Tabla 1 recoge la organización de esas competencias genéricas que ha realizado un grupo de investigadores de la Universidad de Granada (Moreno; Bajo; Moya; Maldonado; Tudela, 2007). Esta clasificación se basa en el carácter más o menos básico de las competencias y en sus aspectos instrumentales ${ }^{4}$. 


\section{Tabla 1 - Clasificación de las Competencias Genéricas Tuning según su Carácter Básico, de Intervención o Específico}

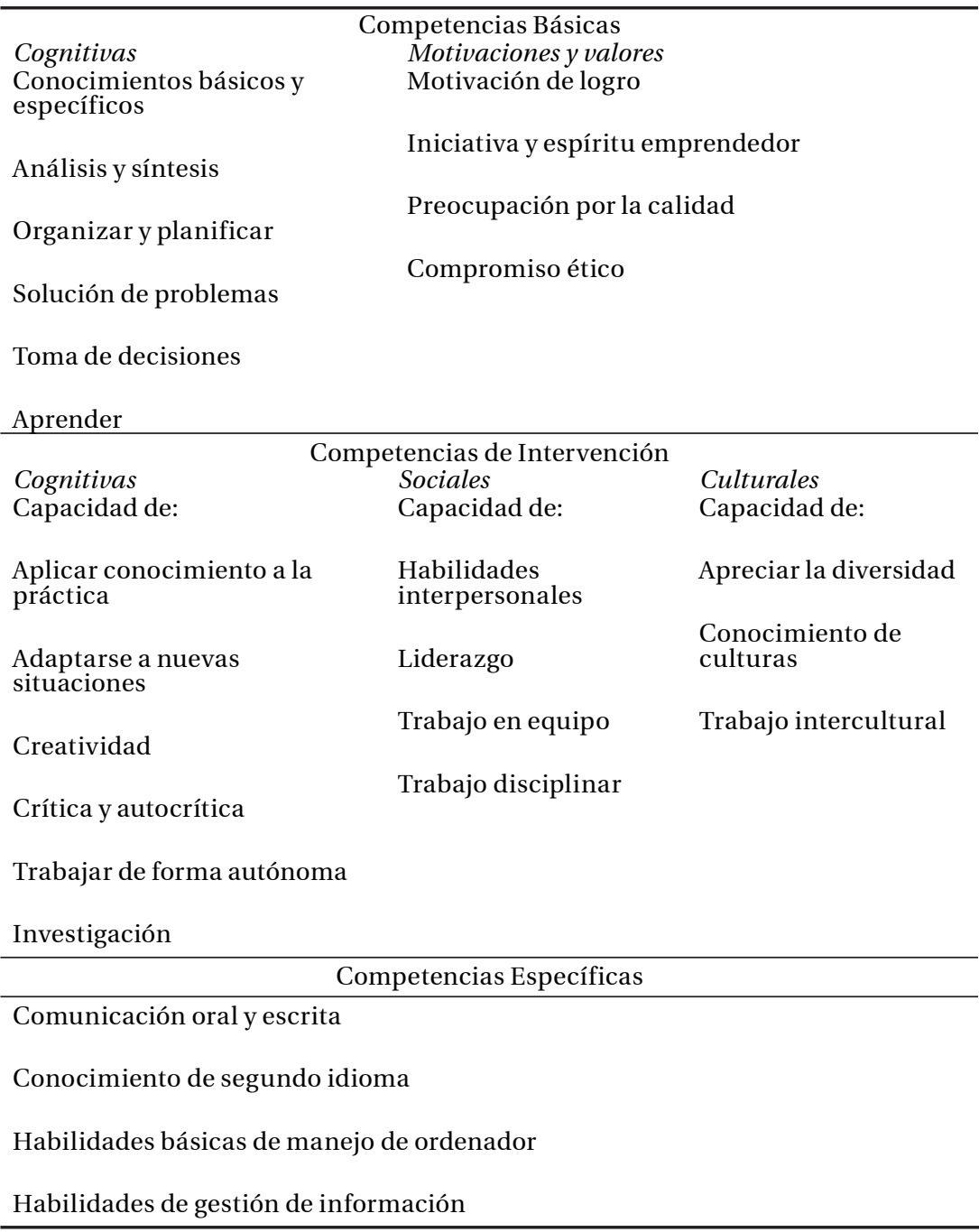

Origen: Moreno; Bajo; Moya; Maldonado; Tudela, 2007, p. 7.

La principal contribución del trabajo de Moreno y sus colaboradores reside en que han elaborado un significado conciso y estructurado para cada una de estas competencias. Del listado anterior destacamos las competencias cognitivas, básicas y de intervención, relevantes para este estudio. También subrayamos las competencias sociales de intervención, singularmente el trabajo disciplinar y el trabajo en equipo. Además, hemos localizado diferentes acercamientos a cómo se pueden

1096 Educação \& Realidade, Porto Alegre, v. 39, n. 4, p. 1089-1111, out./dez. 2014 Disponível em: <http://www.ufrgs.br/edu_realidade> 
diseñar e implementar metodologías que persigan el desarrollo de estas competencias (Fernández, 2006).

Otros trabajos han hecho hincapié en caracterizar algunas de esas competencias en términos de diferentes niveles de dominio, indicadores y descriptores que permiten hacer un seguimiento del grado en el que se desarrollan en programas de formación universitaria (Villa; Poblete, 2007).

Como veremos a continuación, esta estructura de competencias ha sido importante en el establecimiento del marco curricular universitario español para la titulación que forman al profesorado de Matemáticas de Educación primaria.

\section{El Grado de Maestro de Educación Primaria en España}

Uno de los avances del EEES ha consistido en organizar las titulaciones universitarias en términos de Grado y su continuidad en forma de Máster y Doctorado (Ministerio de Educación y Ciencia, 2007b, p. 44038). Desde hace algunos años, existen trabajos que constatan el esfuerzo de diferentes colectivos por operativizar la noción de competencia en este campo de la formación de profesores de Educación primaria. Un ejemplo importante lo constituyen las actividades desarrolladas en diferentes universidades españolas para adecuar progresivamente diversas titulaciones al EEES mediante experiencias piloto (ver ejemplo en Mesa et al. [2007]). Entre esas titulaciones se encuentra la de Maestro: Especialidad de Educación Primaria de la Universidad de Granada; en Lupiáñez, Molina, Flores y Segovia (2007) se ejemplifica uno de estos trabajos en el caso de la formación matemática de los futuros maestros.

Precisamente nos centraremos en las directrices del Plan de Estudios del Grado de Maestro en Educación Primaria que se ha elaborado en la Universidad de Granada (Equipo Docente del Plan de Estudios del Grado de Maestro en Educación Primaria, 2010), ya que en él se han seleccionado las competencias que establecen el perfil profesional para este título. Estas competencias se estructuran en torno a cuatro grupos: generales, específicas del título, específicas de la formación básica y específicas de la optatividad. Las competencias relativas a este último grupo, se detallan en la descripción de las materias que configuran los distintos itinerarios de las menciones calificadoras, por lo que aquí nos centraremos en los tres primeros grupos de competencias.

\section{Competencias Generales}

La selección de las competencias incluidas en este grupo surgen del Libro Blanco de Magisterio (ANECA, 2005a; 2005b) y de la Orden ECI/3857/2007 en la que se establecen los requisitos para la verificación de los títulos universitarios que habilitan para el ejercicio de la profesión de Maestro en Educación primaria (Ministerio de Educación y Ciencia,

Educação \& Realidade, Porto Alegre, v. 39, n. 4, p. 1089-1111, out./dez. 2014.1097

Disponível em: <http://www.ufrgs.br/edu_realidade> 
2007a). En este listado, las competencias se organizan en cuatro apartados, siguiendo la estructura propuesta en el marco del proyecto Tuning que hemos citado antes.

\section{Instrumentales}

CG 1. Analizar y sintetizar la información.

CG 2. Organizar y planificar el trabajo.

CG 3. Identificar, formular e investigar problemas.

CG 4. Examinar alternativas y tomar decisiones.

CG 5. Comunicar oralmente y por escrito con orden y claridad, en la propia lengua y en una segunda lengua.

CG 6. Buscar, seleccionar, utilizar y presentar la información usando medios tecnológicos avanzados

\section{Personales}

CG 7. Adquirir y desarrollar habilidades de relación interpersonal.

CG 8. Trabajar en equipo y comunicarse en grupos multidisciplinares.

CG 9. Expresar y aceptar la crítica.

CG 10. Apreciar la diversidad social y cultural, en el marco del respeto de los Derechos Humanos y la cooperación internacional.

CG 11. Fomentar y garantizar los principios de accesibilidad universal, igualdad, no discriminación y los valores democráticos y de la cultura de paz.

CG 12. Desempeñar su trabajo con compromiso ético hacia sí mismo y hacia los demás.

\section{Sistémicas}

CG 13. Investigar y seguir aprendiendo con autonomía.

CG 14. Innovar con creatividad.

CG 15. Trabajar de forma autónoma y liderar equipos.

CG 16. Diseñar y gestionar proyectos e iniciativas para llevarlos a cabo.

CG 17. Afrontar los retos personales y laborales con responsabilidad, seguridad, voluntad de autosuperación y capacidad autocrítica.

CG 18. Mostrar compromiso ético con los temas medioambientales.

\section{Disciplinares y Profesionales}

CG 19. Comprender y relacionar los conocimientos generales y especializados propios de la profesión teniendo en cuenta tanto su singularidad epistemológica como la especificidad de su didáctica.

1098 Educação \& Realidade, Porto Alegre, v. 39, n. 4, p. 1089-1111, out./dez. 2014. Disponível em: <http://www.ufrgs.br/edu_realidade> 
CG 20. Concebir la profesión docente como un proceso de aprendizaje permanente adaptándose a los cambios científicos, pedagógicos y sociales a lo largo de la vida y comprometido con la innovación, la calidad de la enseñanza y la renovación de prácticas docentes, incorporando procesos de reflexión en la acción y la aplicación contextualizada de experiencias y programas de validez bien fundamentada.

CG 21. Comprender la complejidad de los procesos educativos en general y de los procesos de enseñanza-aprendizaje en particular.

CG 22. Conocer los fundamentos científicos y didácticos de cada una de las áreas y las competencias curriculares de la Educación Primaria: su proceso de construcción, sus principales esquemas de conocimiento, la relación interdisciplinar entre ellas, los criterios de evaluación y el cuerpo de conocimientos didácticos en relación con los procedimientos de enseñanza y aprendizaje respectivos.

CG 23. Conocer los fundamentos psicológicos, pedagógicos y sociales de los procesos de desarrollo y aprendizaje en los diversos contextos educativos

CG 24. Diseñar, planificar, investigar y evaluar procesos educativos individualmente y en equipo.

CG 25. Abordar con eficacia situaciones de aprendizaje de lenguas en contextos multiculturales y plurilingües.

CG 26. Fomentar en el alumnado hábitos lectores y el análisis crítico de textos de los diversos dominios científicos y humanísticos incluidos en el currículo escolar.

CG 27. Diseñar y gestionar espacios e intervenciones educativas en contextos de diversidad que atiendan a la igualdad de género, la equidad y el respeto a los derechos humanos como valores de una sociedad plural.

CG 28. Generar y mantener un clima positivo de convivencia escolar basado en el respeto a las diferencias individuales, en las relaciones interpersonales y en la participación democrática en la vida del aula y del centro, así como afrontar de forma colaborativa situaciones problemáticas y conflictos interpersonales de naturaleza diversa.

CG 29. Adquirir destrezas, estrategias y hábitos de aprendizaje autónomo y cooperativo y promoverlos entre los estudiantes, estimulando el esfuerzo personal y colectivo.

CG 30. Conocer la organización de los colegios de Educación Primaria y la diversidad de acciones que comprende su funcionamiento, así como los modelos de evaluación y mejora de la calidad de los centros educativos, analizando críticamente sus resultados.

CG 31. Desempeñar adecuadamente las funciones de orientación y tutoría con los estudiantes y sus familias.

CG 32. Colaborar en la detección, diagnóstico y evaluación de las necesidades educativas del alumnado y asumir la programación y puesta en práctica de las medidas de atención a la diversidad que correspondan.

Educação \& Realidade, Porto Alegre, v. 39, n. 4, p. 1089-1111, out./dez. 2014. 1099 Disponível em: <http://www.ufrgs.br/edu_realidade> 
CG 33. Promover la educación democrática para una ciudadanía activa y una cultura de paz, colaborando con los distintos sectores de la comunidad educativa y el entorno social.

CG 34. Mantener una actitud crítica y autónoma en relación con los saberes, valores y prácticas que promueven las instituciones sociales valorando especialmente el papel de la ciencia y la tecnología en la sociedad, así como la importancia de una sólida formación humanística.

CG 35. Conocer y aplicar en las actividades de aula las tecnologías de la información y la comunicación, para impulsar un aprendizaje comprensivo y crítico. Discernir selectivamente la información audiovisual que contribuya a los aprendizajes, a la formación cívica y a la riqueza cultural.

CG 36. Conocer las funciones, posibilidades y limitaciones de la educación para afrontar las responsabilidades sociales, promoviendo alternativas que den respuestas a dichas necesidades, en orden a la consecución de un futuro solidario y sostenible.

\section{Competencias Específicas del Título}

Las competencias que los futuros maestros deben desarrollar son las siguientes:

C1. Conocer las áreas curriculares de la Educación Primaria, la relación interdisciplinar entre ellas, los criterios de evaluación y el cuerpo de conocimientos didácticos en torno a los procedimientos de enseñanza y aprendizaje respectivos.

C2. Diseñar, planificar y evaluar procesos de enseñanza y aprendizaje, tanto individualmente como en colaboración con otros docentes y profesionales del centro.

C3. Abordar con eficacia situaciones de aprendizaje de lenguas en contextos multiculturales y plurilingües. Fomentar la lectura y el comentario crítico de textos de los diversos dominios científicos y culturales contenidos en el currículo escolar.

C4. Diseñar y regular espacios de aprendizaje en contextos de diversidad y que atiendan a la igualdad de género, a la equidad y al respeto a los derechos humanos que conformen los valores de la formación ciudadana.

C5. Fomentar la convivencia en el aula y fuera de ella, resolver problemas de disciplina y contribuir a la resolución pacífica de conflictos. Estimular y valorar el esfuerzo, la constancia y la disciplina personal en los estudiantes.

C6. Conocer la organización de los colegios de educación primaria y la diversidad de acciones que comprende su funcionamiento. Desempeñar las funciones de tutoría y de orientación con los estudiantes y sus familias, atendiendo las singulares necesidades educativas de los

1100 Educação \& Realidade, Porto Alegre, v. 39, n. 4, p. 1089-1111, out./dez. 2014 Disponível em: <http://www.ufrgs.br/edu_realidade> 
estudiantes. Asumir que el ejercicio de la función docente ha de ir perfeccionándose y adaptándose a los cambios científicos, pedagógicos y sociales a lo largo de la vida.

C7. Colaborar con los distintos sectores de la comunidad educativa y del entorno social. Asumir la dimensión educadora de la función docente y fomentar la educación democrática para una ciudadanía activa.

C8. Mantener una relación crítica y autónoma respecto de los saberes, los valores y las instituciones sociales públicas y privadas.

C9. Valorar la responsabilidad individual y colectiva en la consecución de un futuro sostenible.

C10. Reflexionar sobre las prácticas de aula para innovar y mejorar la labor docente. Adquirir hábitos y destrezas para el aprendizaje autónomo y cooperativo y promoverlo entre los estudiantes.

C11. Conocer y aplicar en las aulas las tecnologías de la información y de la comunicación. Discernir selectivamente la información audiovisual que contribuya a los aprendizajes, a la formación cívica y a la riqueza cultural.

C12. Comprender la función, las posibilidades y los límites de la educación en la sociedad actual y las competencias fundamentales que afectan a los colegios de educación primaria y a sus profesionales. Conocer modelos de mejora de la calidad con aplicación a los centros educativos.

\section{Competencias Específicas de Formación Básica}

Las competencias específicas de la titulación de Maestro se definen en el Anexo II de la Orden ECI/3857/2007 (Ministerio de Educación y Ciencia, 2007a), por lo que son comunes a todas las universidades españolas. Estas competencias se organizan en diez módulos correspondientes a diferentes áreas de conocimiento. Las relativas a Matemáticas se detallan en el sexto módulo y son las siguientes:

CDM6.1. Adquirir competencias matemáticas básicas (numéricas, cálculo, geométricas, representaciones especiales, estimación y medida, organización e interpretación de la información, etc.).

CDM6.2. Conocer el currículo escolar de matemáticas.

CDM6.3. Analizar, razonar y comunicar propuestas matemáticas.

CDM6.4. Plantear y resolver problemas vinculados con la vida cotidiana.

CDM6.5. Valorar la relación entre matemáticas y ciencias como uno de los pilares del pensamiento científico.

CDM6.6. Desarrollar y evaluar contenidos del currículo mediante recursos didácticos apropiados y promover las competencias correspondientes en los estudiantes.

En las competencias ejemplificadas, vemos ideas que se reiteran con diferente precisión y énfasis y que subrayan que los conocimientos

Educação \& Realidade, Porto Alegre, v. 39, n. 4, p. 1089-1111, out./dez. 2014.1101 Disponível em: <http://www.ufrgs.br/edu_realidade> 
profesionales requeridos para ser profesor de una o de varias disciplinas ${ }^{5}$ demandan donde se:

\begin{abstract}
[...] destacan unos determinados conocimientos, conceptuales y procedimentales, que se centran, en cada caso, en los contenidos disciplinares y en los conocimientos epistemológicos y didácticos propios de cada materia, en los conocimientos sobre planificación y organización del trabajo escolar, sobre el análisis y síntesis didácticos de los contenidos disciplinares, sobre el papel de la resolución de problemas en la disciplina, así como de las estrategias para la toma de decisiones, diseño de tareas e instrumentos de evaluación (Lupiáñez, 2009, p. 149).
\end{abstract}

Este extenso listado pone de manifiesto la complejidad del establecimiento de los requerimientos profesionales que se exige a los profesores de Educación primaria. Aunque todas las competencias son importantes para el desarrollo de la labor docente de los maestros, en nuestro caso cobran especial importancia las referidas a matemáticas. En Ruiz, Molina, Lupiáñez, Segovia y Flores (2009) y en Segovia y Rico (2011), por ejemplo, mostramos el modo en el que concretamos esas competencias en el primer curso de formación de maestros en el área de matemáticas en la Universidad de Granada.

Profundizando en esas competencias, queremos finalmente destacar dos acercamientos centrados en la matemática, si bien no específicamente para Educación primaria.

\title{
Competencias del Profesor de Matemáticas
}

Continuando la reflexión anterior acerca de los requerimientos que plantean las competencias convenidas para los profesores, nos centramos ahora en dos acercamientos recientes, sobre el establecimiento de las competencias profesionales del profesor de matemáticas. En primer lugar presentamos las reflexiones de Moguen Niss sobre las competencias del profesor de matemáticas. Consideramos relevantes estas aportaciones, que dan continuidad y se basan en el trabajo desarrollado por este autor para las competencias matemáticas escolares, que están en la base del estudio PISA (OCDE, 2005). En segundo lugar nos centraremos en algunos de los resultados y acuerdos alcanzados en el Seminario Itermat, celebrado en España en 2004, que ha sido, hasta el momento, la principal aportación española con repercusión institucional para el establecimiento de las competencias específicas del profesor de matemáticas. Destacamos esta aportación ya que los resultados acordados, aunque se refieren a profesores de Educación secundaria, brindan sin duda un marco de reflexión válido también para el caso de maestros.

1102 Educação \& Realidade, Porto Alegre, v. 39, n. 4, p. 1089-1111, out./dez. 2014 Disponível em: <http://www.ufrgs.br/edu_realidade> 


\section{¿Qué Significa ser un Profesor de Matemáticas Competente?}

En el marco del Danish KOM Project ${ }^{6}$, además de investigar sobre la clasificación y organización de las competencias matemáticas de los escolares, también se produjeron resultados acerca de cómo debe orientar su actividad docente un profesor de cara a desarrollar esas competencias en sus alumnos (Niss, 2004a; 2004b).

La pregunta que aparece en el título de este epígrafe la plantea el propio Niss y la responde sosteniendo que un profesor de matemáticas competente es aquel que "[...] de una manera efectiva y eficiente es capaz de ayudar a sus escolares a construir y desarrollar competencias matemáticas" (Niss, 2006, p. 44).

Esta caracterización de profesor competente posee al menos dos implicaciones. En primer lugar, el profesor debe poseer un dominio de las competencias matemáticas que serán objeto de aprendizaje por parte de sus escolares que vaya acorde con el nivel educativo en el que se centra su actividad docente. Esto implica que el profesor de matemáticas en formación necesita desarrollar y lograr cierta maestría sobre una serie de conocimientos matemáticos, capacidades, destrezas y actitudes hacia las matemáticas y que debe poder utilizarlos en una variedad de contextos y situaciones propios de un ciudadano alfabetizado.

Si consideramos las ocho competencias que desglosan la competencia matemática general del estudio PISA (OCDE, 2005), podemos sostener que entre los conocimientos, destrezas y capacidades necesarias para el profesor de matemáticas, siempre en relación con cada uno de los temas que forman parte del currículo de secundaria, se encuentran (Lupiáñez, 2009, p. 153-154):

- El manejo de los principales conceptos y procedimientos matemáticos (pensar y razonar).

- Los procedimientos y técnicas usuales de prueba y demostración (argumentar y justificar).

- La capacidad de expresar ordenadamente y con claridad las propias ideas matemáticas (comunicar).

- La selección de tareas que pongan en juego diferentes fases del proceso de modelización (modelizar).

- La sección y secuenciación de problemas en diferentes situaciones y con distintos niveles de complejidad (plantear $y$ resolver problemas).

- Los distintos sistemas de representación para un mismo concepto y la capacidad para expresar sus propiedades en cada uno de ellos (representar).

- Emplear de manera operativa el simbolismo matemático y poner en juego los rudimentos y las destrezas básicas (usar el lenguaje simbólico, formal y técnico y las operaciones).

- La capacidad de usar recursos tecnológicos que fomenten y apoyen el aprendizaje (emplear soportes y herramientas tecnológicas).

Educação \& Realidade, Porto Alegre, v. 39, n. 4, p. 1089-1111, out./dez. 2014.1103

Disponível em: <http://www.ufrgs.br/edu_realidade> 
Todos estos conocimientos y capacidades muestran la necesidad de un considerable dominio de los contenidos del programa escolar de matemáticas, centrado en las estructuras conceptuales, en el uso experto de los correspondientes sistemas de representación y en el manejo de los diversos contextos y situaciones en los que tales contenidos tienen un uso social convenido, así como en la aplicación de diversas estrategias para la resolución de problemas.

Estos conocimientos, destrezas y capacidades constituyen el núcleo del análisis de contenido (Gómez, 2007). Este procedimiento, como parte del análisis didáctico ${ }^{7}$, incluye un estudio detallado de cada estructura conceptual en términos de conocimientos conceptuales y procedimentales, de los sistemas de representación de cada tópico y su correspondiente análisis fenomenológico, que son los distintos componentes del análisis de contenido, constituyen parte sustantiva del conocimiento profesional en el dominio conceptual requerido para la formación inicial del profesor de matemáticas (Lupiáñez, 2013).

Al profesor en formación le es imprescindible desarrollar su propia competencia matemática, pero no le resulta suficiente. Por ello, en segundo término, Niss también afirma que un profesor de matemáticas competente debe poseer seis competencias didácticas y pedagógicas centradas en matemáticas, que son las siguientes (Niss, 2006, p. 44-45):

Competencia curricular: centrada en analizar, evaluar, relacionar e implementar programas formativos y currículos existentes, así como construir otros nuevos.

Competencia de enseñanza: para diseñar, planificar, organizar, orquestar y gestionar la enseñanza de las matemáticas como por ejemplo crear un amplio espectro de situaciones de enseñanza y aprendizaje, inspirar y motivar a los escolares o discutir con ellos sobre currículos o sobre esas actividades de enseñanza y aprendizaje.

Competencia sobre aprendizaje: centrada en descubrir, interpretar y analizar el aprendizaje de las matemáticas de los escolares, así como sus nociones, creencias y actitudes hacia las matemáticas. También incluye identificar el desarrollo individual de cada uno de los escolares.

Competencia sobre evaluación: para identificar, evaluar, caracterizar y comunicar los resultados de los escolares y sus competencias. También incluye seleccionar, modificar, construir, analizar críticamente e implementar una variedad de formas de evaluación e instrumentos para diferentes propósitos formativos y sumatorios.

Competencia colaborativa: centrada en colaborar con diferentes colegas de matemáticas y de otras disciplinas, así como con otras personas relacionadas con la enseñanza de las matemáticas y sus condicionantes, como padres o autoridades.

Competencia para el desarrollo profesional: para desarrollar la propia competencia como profesor de matemá-

1104 Educação \& Realidade, Porto Alegre, v. 39, n. 4, p. 1089-1111, out./dez. 2014 Disponível em: <http://www.ufrgs.br/edu_realidade> 
ticas (meta-competencia), y que incluye, participar y relacionarse con actividades de desarrollo profesional, tales como cursos formativos, proyectos o conferencias; reflexionar sobre la propia enseñanza y sus necesidades, y mantenerse actualizado acerca de nuevas tendencias en la investigación y en la práctica.

Niss concluye señalando que en un programa de formación inicial de profesores de matemáticas, la Didáctica de la Matemáticas constituye un componente crucial para iniciar el desarrollo de las cuatro primeras competencias descritas antes, mientras que las dos últimas, se desarrollan con motivo de la práctica docente (Niss, 2006).

A la competencia curricular inicial la consideramos como una competencia general, que engloba las cuatro anteriores y que requiere de conocimientos generales sobre el currículo de matemáticas, sus finalidades, sus niveles y dimensiones, su organización; sobre historia de la matemática y de la educación matemática; sobre las matemáticas escolares y sus fuentes epistemológicas y axiológicas; sobre psicología de la educación, teorías del aprendizaje escolar y su funcionalidad en el aprendizaje de las matemáticas; sobre teorías de la enseñanza e instrucción y su funcionalidad en la enseñanza de las matemáticas; sobre marcos teóricos, principios, modalidades e instrumentos de evaluación. Todos estos conocimientos, capacidades y destrezas asociados constituyen los principios que articulan un conocimiento general y básico sobre Didáctica de la Matemática, desarrollado extensamente en Rico (1992; 1997a) y Rico y Gutiérrez (1994). En este trabajo no consideramos las dos competencias del nivel profesional señaladas por Niss, por centrarnos en un programa de formación inicial.

\section{Conocimiento General Básico y Conocimiento Básico de la Profesión del Profesor de Matemáticas}

En el Seminario Itermat (Recio, 2004), y dentro del contexto del proyecto Tuning, se analizaron las nociones de conocimiento general básico (CGB) y conocimiento básico de la profesión (CBP) (Bajo; Maldonado; Moreno; Moya; Tudela, 2003; González; Wagenaar, 2003). Dentro del contexto de la definición de competencias para la Licenciatura de Matemáticas, González (2004, p. 4) interpreta esas nociones de la siguiente manera:

El CGB corresponde a fundamentos disciplinares generales de la Didáctica de la Matemática que son referentes teóricos para el profesor de matemáticas. El CBP es el que capacita para investigar/ejercer la práctica de la profesión de profesor de matemáticas de modo independiente. Esta separación no tiene fronteras claras en los planes de formación de profesores. Las investigaciones sobre la naturaleza del conocimiento del profesor de matemáticas

Educação \& Realidade, Porto Alegre, v. 39, n. 4, p. 1089-1111, out./dez. 2014.1105 Disponível em: <http://www.ufrgs.br/edu_realidade> 
sitúan a este conocimiento en un plano intermedio entre lo teórico y lo empírico y lo describen como un sistema de funcionamiento integrado que combina saberes de naturaleza académica y su puesta en práctica.

Llinares (2004, p. 2) propone que la discusión sobre las competencias del profesor de matemáticas se articule en torno a tres "sistemas de actividad”: (a) organizar el contenido matemático para enseñarlo; (b) analizar e interpretar las producciones matemáticas de los alumnos; y (c) gestionar el contenido matemático en el aula.

Uno de los acuerdos de esa reunión fue el establecimiento de las competencias básicas del profesor de matemáticas de secundaria, entre las que destacan (Rico, 2004, p. 8-9):

1. El dominio de los contenidos matemáticos de Educación Secundaria desde una perspectiva matemática superior y su conocimiento como objetos de enseñanza-aprendizaje;

2. la organización curricular y planificación de estos contenidos matemáticos para su enseñanza;

3. el análisis, interpretación y evaluación de los conocimientos matemáticos de los alumnos a través de sus actuaciones y producciones matemáticas; $\mathrm{y}$

4. la capacidad de gestión del contenido matemático en el aula.

Como hemos señalado antes, estos trabajos centrados en competencias del profesorado de Educación secundaria, tienen una lectura posible y relevante en términos de las competencias del maestro. Por una parte, estas competencias enfatizan un conocimiento genérico y especializado, científico y técnico de matemáticas, sobre sus contenidos, métodos y estrategias de resolución de problemas, que resultan fundamentales para la enseñanza de las matemáticas en Educación primaria.

También se expresan competencias sobre diseño y planificación que permitan organizar y reformular los procesos de enseñanza-aprendizaje. Capacidades instrumentales como analizar y sintetizar, organizar y proponer problemas, junto con habilidades interpersonales como capacidad para la crítica y para trabajar en grupo son también claves en la formación del maestro, tal y como hemos mostrado en propuestas anteriores.

Finalmente, también se proponen competencias didácticas que consideran los propios conocimientos disciplinares como objetos de enseñanza y aprendizaje, orientadas a la implementación docente y evaluación de los aprendizajes. La capacidad para el análisis didáctico de los contenidos curriculares establece la estructura conceptual de una determinada técnica o teoría y el modo de abordarla para su comprensión y aprendizaje. En todos estos casos, se expresan expectativas de aprendizaje importantes para los profesores de Educación primaria.

1106 Educação \& Realidade, Porto Alegre, v. 39, n. 4, p. 1089-1111, out./dez. 2014. Disponível em: <http://www.ufrgs.br/edu_realidade> 


\section{Una Selección Fundada de la Formación del Maestro}

Al inicio de este documento hemos acotado una caracterización de la noción de competencia en el caso de los futuros profesores. Después, hemos descrito y analizado tres fuentes principales que han brindado diferentes propuestas de competencias del profesor: las que surgen de los proyectos y las innovaciones de la Comunidad Europea, las establecidas en el Grado de Maestro de Educación Primaria y, finalmente, dos listados de competencias centrados en Matemáticas.

La posición que hemos asumido de la noción de competencia, que se sustenta en una visión funcional de la formación inicial (Lupiáñez, 2009; Rico; Lupiáñez, 2008), considera la activación de unos determinados procesos cognitivos sobre algunos conocimientos para dar respuesta a problemas o cuestiones en diferentes situaciones y contextos. Estos componentes se identifican en las tres propuestas analizadas, enfatizando su fundamento conceptual y reforzando, por tanto, la relevancia y coherencia de nuestra selección.

Además, en todos los casos las propuestas vienen avaladas y respaldadas institucionalmente por diferentes estamentos. Desde políticas de intervención europeas, a investigaciones realizadas por especialistas de reconocido prestigio, pasando por los avances logrados por algunas universidades españolas, en cada caso las competencias señaladas provienen de una reflexión institucional y colegiada que refuerzan su importancia y su reconocimiento.

Finalmente, es destacable el carácter finito, interrelacionado y jerárquico de los listados de competencias que hemos remarcado. Esta característica permite que las propuestas tengan una operatividad notoria para el diseño, puesta en práctica y evaluación de programas formativos, ya que facilitan el establecimiento organizado y estructural de las expectativas formativas que deben perseguirse en esos programas, sugieren posibles orientaciones metodológicas y de evaluación de aprendizajes, además de indicar las diferentes facetas de los programas que pueden ser objeto de evaluación y mejora.

Recebido em 24 de março de 2014 Aprovado em 10 de junho de 2014

\section{Notas}

1 Achtenhagen, Oser y Renold citan dos fuentes pero solo referencian una de ellas. Afirman que esa conceptualización de la competencia del profesor proviene de los trabajos de Heinrich Roth y Lotear Reetz, y que fue adaptada en el marco de un proyecto PISA sobre formación profesional (Baethge; Achtenhagen; Arends; Babic; Baethge-Kinsky; Weber, 2006).

2 Es posible consultar todo el recorrido y la evolución del EEES desde su origen hasta la actualidad en: <http://www.eees.es/es/documentacion/>.

Educação \& Realidade, Porto Alegre, v. 39, n. 4, p. 1089-1111, out./dez. 2014.1107 Disponível em: <http://www.ufrgs.br/edu_realidade> 
3 No entramos en detalle en la descripción de Proyecto Tuning América Latina por varias razones. En primer lugar este proyecto no se sustenta en un marco político unificado como en el caso de Europa, porque en América Latina no existe ese marco y porque están aún en un proceso de integración regional (p. 19). En nuestro caso, el marco político de Bolonia contextualiza todas las reformas curriculares en Educación Superior.

4 En el caso de Tuning América Latina, no se han establecido listados de competencias genéricas muy diferentes con esta propuesta europea, sino que se produce una reorganización de estas y se añaden tres nuevas: “[...] responsabilidad social y compromiso ciudadano, compromiso con la preservación del medio ambiente y compromiso con su medio cultural" (Beneitone et al., 2007, p. 45).

5 En Lupiáñez (2009, p. 142-149), se analizan las competencias propuestas en otras titulaciones relacionadas con la docencia.

6 Este proyecto fue promovido por el Ministerio de Educación de Dinamarca y otros estamentos, con el objetivo de crear una plataforma para llevar a cabo una profunda reforma de la educación matemática en ese país, desde la educación básica hasta la universitaria (Niss, 2003). KOM son las siglas en danés de Competencias y Aprendizaje de las Matemáticas.

7 Procedimiento para, entre otras funciones, el diseño, puesta en práctica y evaluación de actividades de enseñanza y aprendizaje (Rico, 2013).

\section{Referencias}

ABBOTT, Ian; HUDDLESTON, Prue. Standards, Competence and Knowledge: Initial Teacher Training and Business. International Journal of Value-Based Management, v. 13, p. 215-227, 2000.

ACHTENHAGEN, Frank; OSER, Fritz; RENOLD, Ursula. Epilogue. In: ACHTENHAGEN, Frank; OSER, Fritz; RENOLD, Ursula (Org.). Competence Oriented Teacher Training. Rotterdam: Sense Publisher, 2006. P. 297-304.

ALLEGRE, Claude; BERLINGUER, Luigi; BLACKSTONE, Tessa; RÜTTGERS, Jürgen. Sorbonne Joint declaration. Joint declaration on Harmonisation of the Architecture of the European higher Education System. 1998. Disponible en: <http://www.eees.es/pdf/Sorbona_EN.pdf/>. Descargado el: 17 abr. 2009.

ANECA. Libro Blanco: Título de Grado en Magisterio (v. 1). 2005a. Disponible en: <http://www.aneca.es/activin/docs/libroblanco_jun05_magisteriol.pdf/>. Descargado el: 26 mar. 2008.

ANECA. Libro Blanco: Título de Grado en Magisterio (v. 2). 2005b. Disponible en: <http://www.aneca.es/activin/docs/libroblanco_jun05_magisterio2.pdf/>. Descargado el: 26 mar. 2008.

BAETHGE, Martin; ACHTENHAGEN, Frank; ARENDS, Lena; BABIC, Edvin; BAETHGE-KINSKY, Volker; WEBER, Susanne. PISA-VET: A Feasibility-Study. Stuttgart: Franz Steiner Verlag, 2006.

BAJO, Maria Teresa; MALDONADO, Antonio; MORENO, Sergio; MOYA, Miguel; TUDELA, Pio. Las Competencias en el Nuevo Paradigma Educativo para Europa. Granada: Universidad de Granada, 2003.

BECK, Clive; HART, Doug; KOSNIK, Clare. The teaching standards movement and current teaching practices. Canadian Journal of Education, Charlottetown, v. 27, n. 2-3, p. 175-194, 2002.

1108 Educação \& Realidade, Porto Alegre, v. 39, n. 4, p. 1089-1111, out./dez. 2014 Disponível em: <http://www.ufrgs.br/edu_realidade> 
BENEITONE, Pablo; ESQUETINI, César; GONZÁLEZ, Julia; MALETÁ, Maida M.; SIUFI, Gabriela; WAGENAAR, Robert. Reflexiones y Perspectivas de la Educación Superior en América Latina: informe final Proyecto Tuning América Latina 2004-2007. Bilbao: Universidad de Deusto, Universidad de Groningen, 2007.

DELORS, Jacques. La Educación encierra un Tesoro: informe a la UNESCO de la Comisión Internacional sobre la educación para el siglo XXI. Madrid: Santillana, 1996.

DEPARTMENT OF EDUCATION AND TRAINING. Competency Framework for Teachers. East Perth: Autor, 2004.

EINEM, Caspar; SCHMIT, Gerard; ADE, Jan; TOTOMANOVA, Anna Mmia; ZEMAN, Eduard; VESTAGER, Margrethe et al. El Espacio Europeo de la Enseñanza Superior: declaración conjunta de los ministros europeos de educación reunidos en Bolonia el 19 de junio de 1999. 1999. Disponible en: <http://www.eees.es/ pdf/Bolonia_ES.pdf/>. Descargado el: 17 abr. 2009.

EQUIPO DOCENTE DEL PLAN DE ESTUDIOS DEL GRADO DE MAESTRO EN EDUCACIÓN PRIMARIA. Plan de Estudios del Grado de Maestro en Educación Primaria. 2010. Disponible en: <http://grados.ugr.es/ed_primaria/pages/titulacion/objetivos>. Descargado el: 16 abr. 2010.

FERNÁNDEZ, Amparo. Metodologías Activas para la Formación de Competencias. Educatio siglo XXI, Murcia, v. 24, p. 35-56, 2006.

GÓMEZ, Pedro. Análisis Didáctico y Diseño Curricular en Matemáticas. Revista EMA, Bogotá, v. 7, n. 3, p. 251-293, 2002.

GÓMEZ, Pedro. Desarrollo del Conocimiento Didáctico en un Plan de Formación Inicial de Profesores de Matemáticas de Secundaria. Universidad de Granada, 2007.

GONZÁLEZ, Julia; WAGENAAR, Robert. Tuning Educational Structures in Europe. Informe final. Fase uno. Bilbao: Universidad de Deusto y Universidad de Groningen, 2003.

GONZÁLEZ, Julia; WAGENAAR, Robert. Tuning Educational Structures in Europe. Informe final. Fase dos. Bilbao: Universidad de Deusto y Universidad de Groningen, 2006.

GONZÁLEZ, María José. Contribución de la Opción Educativa a las Competencias del Licenciado en Matemáticas. 2004. Trabajo presentado en Seminario Itermat, organizado por ICMI-E y Universidad de Granada. Disponible en: <http://www.ugr.es/ vic_plan/formacion/itermat/Program/221S3.htm/>. Descargado el: 7 jan. 2005.

LLINARES, Salvador. La Actividad de Enseñar matemáticas como Organizador de la Formación Inicial de Profesores de Matemáticas de Secundaria. Adecuación al itinerario educativo del Grado de Matemáticas. Trabajo presentado en Itinerario Educativo de la Licenciatura de Matemáticas, Granada, 2004.

LUENGO, Julían; LUZÓN, Antonio; TORRES, Monica. El Enfoque por Competencias en el Desarrollo de Políticas de Formación del Profesorado. Entrevista a Claude Lessard. Profesorado. Revista de Curriculum y Formación del Profesorado, Granada, v. 12, n. 3, p. 1-16, 2008.

LUPIÁÑEZ, José Luis. Expectativas de Aprendizaje y Planificación Curricular en un Programa de Formación Inicial de Profesores de Matemáticas de Secundaria. Universidad de Granada, 2009.

LUPIÁÑEZ, José Luis. Análisis Didáctico: la planificación del aprendizaje desde una perspectiva curricular. In: RICO, Luis; LUPIÁÑEZ, José Luis; MOLINA,

Educação \& Realidade, Porto Alegre, v. 39, n. 4, p. 1089-1111, out./dez. 2014.1109

Disponível em: <http://www.ufrgs.br/edu_realidade> 
Marta (Org.). Análisis Didáctico en Educación Matemática: metodología de investigación, formación de profesores e innovación curricular. Granada: Editorial Comares, 2013. P. 81-101.

LUPIÁÑEZ, José Luis; MOLINA, Marta; FLORES, Pablo; SEGOVIA Isidoro. Mathematics Primary Teacher Training in the Context of the European Higher Education Area. The International Journal of Interdisciplinary Social Sciences, v. 2, n. 4, p. 223-232, 2007.

MESA, C.; GARCÍA, E.; GONZÁLEZ, Carlos; GONZÁLEZ, Y.; PEÑALVER, M.; INIESTA, M. A. et al. In: II JORNADAS DE TRABAJO SOBRE EXPERIENCIAS PILOTO EEES EN LAS UNIVERSIDADES ANDALUZAS. Granada: Comisión EEES de Universidades Andaluzas, 2007.

MINISTERIO DE EDUCACIÓN Y CIENCIA. ORDEN ECI3857/2007, de 27 de diciembre, por la que se establecen los requisitos para la verificación de los títulos universitarios oficiales que habiliten para el ejercicio de la profesión de Maestro en Ecuación Primaria. BOE, 312, 2007a. P. 53747-53750.

MINISTERIO DE EDUCACIÓN Y CIENCIA. Real Decreto 1393/2007, de 29 de octubre, por el que se establece la ordenación de las enseñanzas universitarias oficiales. BOE, 260, 2007b. P. 44037-44048.

MORENO, Sergio; BAJO, Maria Teresa; MOYA, Miguel; MALDONADO, Antonio; TUDELA, Pio. Las Competencias en el Nuevo Paradigma Educativo para Europa. Granada: Vicerrectorado de Planificación, Calidad y Evaluación Docente de la Universidad de Granada, 2007.

NISS, Mogens. Mathematical Competencies and the Learning of Mathematics: The Danish KOM Project. In: GAGATSIS, Athanasios; PAPASTAVRIDES, Stavros (Org.). $3^{\text {rd }}$ Mediterranean Conference on Mathematical Education. Athens: Hellenic Mathematical Society, 2003. P. 115-124.

NISS, Mogens. NCTM Research Catalyst Conference. Trabajo presentado en NCTM Research Catalyst Conference, Reston, VA, 2004a.

NISS, Mogens. The Danish KOM Project and Possible Consequences for Teacher Education. In: STRÄSSER, Rudolf; BRANDELL, Gerd; GREVHOLM, Barbro (Org.). Educating for the Future: proceedings of an international symposium on mathematics teacher education. Göteborg: Royal Swedish Academy of Sciences, 2004b. P. 179-192.

NISS, Mogens. What does it mean to be a Competent Mathematics Teacher? A general problem illustrated by examples from Denmark. In: PRAKTIKA, 230 PANELLENIO SYNEDRIO MATHEMATIKIS PAIDEIAS. Patras, Greece: Elleniki Mathematiki Etaireia, 2006. P. 39-47.

OCDE. Informe PISA 2003: aprender para el mundo del mañana. Madrid: Editorial Santillana, 2005.

OSER, Fritz; ACHTENHAGEN, Frank; RENOLD, Ursula. Competence Oriented Teacher Training: old research demands and new pathways. In: OSER, Fritz; ACHTENHAGEN, Frank; RENOLD, Ursula (Org.). Competence Oriented Teacher Training. Rotterdam: Sense Publisher, 2006. P. 1-7.

RECIO, T. Seminario: Itinerario educativo de la Licenciatura de Matemáticas. Documento de conclusiones y propuestas. La Gaceta de la Real Sociedad Matemática Española, v. 7, n. 1, p. 33-36, 2004.

RICO, Luis. Proyecto Docente. Granada: Universidad de Granada, 1992.

RICO, Luis. Bases Teóricas del Currículo de Matemáticas en Educación Secundaria. Madrid: Síntesis, 1997a.

1110 Educação \& Realidade, Porto Alegre, v. 39, n. 4, p. 1089-1111, out./dez. 2014. Disponível em: <http://www.ufrgs.br/edu_realidade> 
RICO, Luis. Dimensiones y componentes de la noción de currículo. In: RICO, Luis (Org.). Bases Teóricas del Currículo de Matemáticas en Educación Secundaria. Madrid: Síntesis, 1997b. P. 377-414.

RICO, Luis. Reflexiones sobre la Formación Inicial del Profesor de Matemáticas de Secundaria. Profesorado. Revista de Currículum y Formación del Profesorado, v. 8, n. 1, 2004. P. 1-15.

RICO, Luis. Análisis Didáctico y Metodología de Investigación. In: RICO, Luis; LUPIÁÑEZ, José Luis; MOLINA, Marta (Org.). Análisis Didáctico en Educación Matemática: metodología de investigación, formación de profesores e innovación curricular. Granada: Editorial Comares, 2013. P. 1-22.

RICO, Luis; GUTIÉRREZ, José. Formación Científico-didáctica del Profesor de Matemáticas de Secundaria. Granada: Instituto de Ciencias de la Educación, 1994.

RICO, Luis; LUPIÁÑEZ, José Luis. Competencias Matemáticas desde una Perspectiva curricular. Madrid: Alianza Editorial, 2008.

RICO, Luis; LUPIÁÑEZ, José Luis; MOLINA, Marta. Análisis Didáctico en Educación Matemática: metodología de investigación, formación de profesores e innovación curricular. Granada: Editorial Comares, 2013.

RUIZ, Francisco; MOLINA, Marta; LUPIÁÑEZ, José Luis; SEGOVIA, Isidoro; FLORES, Pablo. Mathematics Primary Teacher Training at the University of Granada: an adaptation to the EHEA. Electronic Journal of Research in Educational Psychology, v. 17, p. 425-454, 2009.

SEGOVIA, Isidoro; RICO, Luis. Matemáticas para Maestros de Educación Primaria. Madrid: Ediciones Pirámide, 2011.

SOTELO, Ignacio. Cara y Cruz del Proceso de Bolonia. 2009. Disponible en: <http://www.elpais.com/articulo/opinion/Cara/cruz/proceso/Bolonia/ elpepiopi/20090416elpepiopi_4/Tes/>. Descargado el: 22 abr. 2009.

VILLA, Aurelio; POBLETE, Manuel. Aprendizaje Basado en Competencias. Bilbao: Universidad de Deusto y Editorial Mensajero, 2007.

José Luis Lupiáñez es doctor en Matemáticas por la Universidad de Granada. Es profesor titular de la Universidad de Granada. Investigador en la línea de formación de profesores de matemáticas.

E-mail: lupi@ugr.es 